\title{
¿INCIDE LA ISO 14000 EN EL DESEMPEÑO EXPORTADOR DE LAS EMPRESAS COLOMBIANAS?
}

\section{ARE COLOMBIAN ENTERPRISES AFFECTED IN THEIR EXPORT PERFORMANCE BY THE ISO 14000 STANDARD?}

\author{
Claudia Paola García-Castiblanco ${ }^{1}$, Diana Marcela Díaz-Ariza ${ }^{2}$ iD Carlos Andrés Pinzón-Muñoz $^{3}$ iD \\ ${ }^{1}$ Universitaria Agustiniana. Colombia. Email: claudia.garcia@uniagustiniana.edu.co \\ ${ }^{2}$ Universitaria Agustiniana. Colombia.Email: diana.diaza@uniagustiniana.edu.co \\ ${ }^{3}$ Universitaria Agustiniana. Colombia. Email: carlos.pinzonm@uniagustiniana.edu.co
}

Para citar este artículo: García, C. C., Díaz, A. D. y Pinzón, M. C. (2019). ¿Incide la ISO 14000 en el desempeño exportador de las empresas colombianas?. Clío América, 13(25), 243-254. doi: http://dx.doi.org/10.21676/23897848.3253

Recibido: 20 febrero de 2019

Aceptado: 10 de mayo de 2019

Publicado en línea: mayo 24 de 2019

Palabras clave:

ISO 14000; internacionalizac ión; desarrollo sostenible; comercio internacional.

JEL: Q56; F18; M14.

Keywords: ISO 14000; internationaliza tion; sustainable development; international marketing.

\section{RESUMEN}

Los sistemas de gestión ambiental, como los que certifica la Organización Internacional para la Estandarización - ISO 14000, buscan garantizar el buen manejo de los recursos en las empresas, de forma tal que se establezcan prácticas amigables con el ambiente. Son diversas las razones que pueden motivar a las empresas a desarrollar procesos de certificación, como poder cumplir con estándares internacionales y de esta manera acceder a mercados en el extranjero. Siendo Colombia uno de los países en los que más se ha incrementado la adopción de la certificación ISO 14000, vale la pena peguntar si este proceso les genera a las compañías un aumento en el volumen de sus exportaciones. Esta investigación se realiza en el marco de la teoría de la competitividad ambiental. El estudio es de corte transversal e identifica empresas certificadas y que además son exportadoras, midiendo su comportamiento frente a un grupo de compañías no certificadas mediante una prueba de hipótesis no paramétrica. Como resultado se encuentra que existe una diferencia significativa entre los volúmenes exportados por las empresas certificadas y los de las que no lo están, aunque no se puede afirmar que su éxito en ventas en el extranjero se deba a la ISO 14000.

\section{ABSTRACT}

The environmental management systems, which are certified by the Standardization International organization - ISSO 14000 , are in the search to ensure the appropriate resources management of enterprises to determine environmentally friendly conditions. Be able to fulfill the international standards and thus rendering access to international markets is the reason why factories develop certification process. Colombia is one of the countries where the ISSO 14000 norm has been adopted but, it is worth asking if this process spawn increase exportation volume. For the implementation of this research, the export performance was analyzed between exporting and certified markets and non-certified ones through a parametric hypothesis test. As a result, it was identified that there is a significant difference between the number of exportations by certified markets than the ones that are not. However, the study does not state that the abroad sales leverage is because of ISO 14000 . 


\section{INTRODUCCIÓN}

Una necesidad cada vez más recurrente es la de tomar con responsabilidad procesos productivos amigables con el ambiente que garanticen un uso óptimo de los recursos. Esto ha conllevado a que se diseñen mecanismos que permitan verificar que los procedimientos adoptados son acordes con los estándares internacionales. En este contexto, la Organización Internacional para la Estandarización (ISO por su nombre en inglés) es una de las entidades más reconocidas en cuanto al establecimiento y certificación en la implementación de normas que se deben seguir para el desarrollo de sistemas de gestión ambiental (SGA) en las organizaciones. El éxito de la ISO 14000 consiste en que es aplicable a todo tipo de organización, sin distinción de sector o tamaño.

Más allá de la certificación, un sistema de gestión ambiental puede entenderse como una metodología mediante la cual la organización monta su operación con el objetivo de asegurar la protección ambiental. Para ello es necesario tener claro el nivel de impacto de las actividades que se van a desarrollar, con miras a generar estrategias que limiten su impacto (Rowland-Jones, Pryde y Cresser, 2005). Desde luego, el diseño de la política de cada empresa dependerá del sector y del tipo de recursos que se maneje en los procesos, por lo que las estrategias pueden girar alrededor de prácticas como la adopción de tecnologías más limpias, la eficiencia en el uso de los recursos y del espacio, así como en el diseño de la logística, y la innovación que permita mejorar el ciclo de la vida de los productos (Jänicke, 2008).

La implementación de procesos de certificación es costosa para las empresas, ya que en muchos casos implica la aplicación de cambios en los procesos (más si son inesperados y obligados por la legislación). Es por ello que se espera que la organización reciba algún tipo de beneficio a partir de la certificación. Existe una buena cantidad de estudios que se han enfocado en determinar si la certificación en ISO 14000 incide en el rendimiento financiero de las empresas (Miroshnychenko, Barontini y Testa, 2017; Psomas, Fotopoulos, y Kafetzopoulos, 2011; Neves, Salgado y Beijo, 2017); sin embargo, no se ha indagado con el mismo interés la incidencia sobre las exportaciones. Algunos de los trabajos enfocados en esta área son los de Tambunlertchai, Kontoleon y Khanna (2013) y Bellesi, Lehrer y Tal (2005).

Dentro de las diversas investigaciones que buscan identificar si hay beneficios económicos en la implementación de estrategias verdes, la adopción de SGA o las certificaciones tipo ISO 14000 se encuentran resultados muy diversos. Como explican Chen, Ong y Hsu (2016), tal variedad se debe al empleo de diferentes variables como la metodología establecida, el tiempo que abarca el estudio, los países, el tamaño de la empresa, entre otras.

Específicamente, el tipo de actividad o cuán contaminante esta es resulta ser una variable de interés por parte de los investigadores para determinar el logro de ganancias (Clarkson, Li, Richardson y Vasvari, 2011; Lucas y Noordewier, 2016). Al parecer, las industrias altamente contaminantes son las que mayores posibilidades poseen de incrementar sus rendimientos, eso sí, una vez ha transcurrido un lapso considerable desde la aplicación de la estrategia.

Ahora bien, la aplicación de un SGA o la adquisición de una certificación como la ISO 14000 pueden ser incluso más costosas para las empresas que la simple adopción de las prácticas verdes $\mathrm{y}$, por tanto, poner en duda la viabilidad financiera de implementar este tipo de estrategias. Los resultados de las investigaciones en esta materia son igualmente inconclusos.

Feng, Cai, Wang y Zhang (2016) explican que los SGA pueden ayudar a las empresas a obtener ventajas competitivas aportando oportunidades de ahorro de costes ya que las alientan a establecer una planificación ambiental a lo largo de toda la gama de actividades, desde la adquisición de materias primas, pasando por la fabricación, hasta la distribución de productos para minimizar los costos del ciclo de vida de sus productos y desarrollar nuevos con menores costos. Lo, Yeung y Cheng (2012) afirman a su vez que la implementación de la certificación ISO 14000 dentro de la empresa no necesariamente redunda en beneficios económicos, aunque reconocen que pueden encontrarse impactos positivos sobre la rentabilidad o el Return On Assets (ROA) a partir del segundo año de certificación. Por el contrario, Psomas (como se citó en Neves et al., 2017) encontró que con la adopción de las ISO 14001 las compañías implementaron mecanismos que les permitieron desarrollar su desempeño tanto ambiental como de negocio, redujeron costos, mejoraron su imagen pública y expandieron su mercado.

En últimas, se observa que el impacto económico de la aplicación de prácticas verdes, incluida la adopción de la certificación ISO 14000, puede generar diversos efectos sobre el desempeño financiero, los cuales dependerán del tipo de industria y del tamaño de la empresa, entre otros. Sin embargo, ¿qué pasa con el desempeño exportador si una de las razones que conllevan a la búsqueda de las certificaciones es la posibilidad de ampliar el acceso a mercados? 
Los estudios en este sentido son pocos y, por lo mismo, no muy concluyentes. No obstante, hay un factor común a la mayoría y es la tendencia a proponer que las empresas que tienen su punto de operación en países en desarrollo (Tambunlertchai et al., 2013; Bellesi et al., 2005) van a requerir más de las certificaciones para poder acceder a otros mercados, sobre todo si el objetivo son países con consumidores responsables.

Pekovic y Rollan (2016) validan lo anterior. Pese a que su objeto principal de estudio es la ISO 9000, sus resultados pueden extenderse a la ISO 14000, ya que el planteamiento principal de los autores sugiere que los estándares internacionales demuestran la capacidad de la empresa para satisfacer las expectativas de calidad de los clientes y hacer públicos los atributos no observables, especialmente en contextos en los que los clientes pueden ser sensibles a la reputación de calidad. En este sentido, las certificaciones se convierten en el medio de comunicación que permite la interacción entre productor y consumidores con el ánimo de brindar información sobre los procesos de producción, garantizando que en ellos se cumplieron con lo más altos estándares.

King, Lenox y Terlaak (2005) proponen que las empresas están más motivadas para adoptar certificaciones ISO cuando sus compradores están más distantes físicamente. Esto va de la mano con la creencia de que las certificaciones mejoran la imagen de la empresa, lo que permite igualmente ampliar el acceso a mercados (Seijo-García, Filgueira-Vizoso y MuñozCamacho, 2013; Dragičević y Letunić, 2012).

Por último, en los estudios en los que se ha incluido el tema de las exportaciones se encuentran por ejemplo el de Antonietti y Marzucchi (2014), quienes estiman que sí puede haber un estímulo a las exportaciones al propender a acceder a mercados donde la regulación ambiental es más estricta. Sin embargo, una conclusión diferente es la de González, Sarkis y Adenso-Díaz (2008), quienes no logran hallar relación entre la certificación y el aumento de las exportaciones, al igual que Tambunlertchai et al. (2013).

En algunas investigaciones se encuentra que el nivel de internacionalización de las empresas tiene incidencia sobre el interés por acceder a este tipo de certificaciones. Por ejemplo, Luan, Tien y Chen (2016) determinan que a mayor grado de internacionalización, mayor número de certificaciones buscarán las empresas. Sin embargo, el estudio es claro en establecer que las certificaciones no son la primera alternativa de las empresas, sino que son parte de un proceso que se inicia con la adopción de prácticas amigables con el ambiente, lo que puede ser producto de presiones legales o se hace de manera proactiva para luego sí poder acceder a las certificaciones internacionales como la ISO 14000.

En esta misma línea, Aguilera, Hurtado y Aragón (2012), a través de un estudio desarrollado en España, confirman la asociación entre el grado de internacionalización y la adopción de prácticas verdes. Estos autores explican que encontraron una relación entre el tamaño (cuanto más grandes, más propensas a adoptar estos procesos), la antigüedad (cuanto más antiguas, más propensas a adoptar estos procesos) y la diversidad (cuantos más mercados en el extranjero, más propensas a adoptar estos procesos).

Frente a este contexto, Colombia es un país en desarrollo con una participación modesta en los mercados internacionales, cuyo comercio exterior depende ante todo de la exportación de productos tradicionales a mercados de primer mundo. Según Neves et al. (2017), en Colombia existen desde 1998 políticas gubernamentales en asocio con el Banco Interamericano de Desarrollo (BID) que incentivan a las empresas a buscar certificarse tanto en ISO 9000 como en ISO 14000. Es por ello que a partir de 2002 hay un aumento considerado en el número de certificaciones en el país. De esta manera los autores concluyen que en Colombia los factores socioculturales son los que llevan a las empresas a la certificación más que los factores económicos, como el crecimiento en las exportaciones.

La presente investigación tiene por tanto el objetivo de determinar si las empresas colombianas con certificación ISO 14000 han mostrado un mejor desempeño exportador que las no certificadas. Encontrar una respuesta positiva a este planteamiento permitirá atraer a nuevas compañías a la responsabilidad social ambiental.

Este documento está dividido en seis partes: la presente, que corresponde a la introducción; se continúa con una breve explicación de lo que es la ISO y la ISO 14000 con el objetivo de contextualizar al lector; en tercer lugar se aborda el marco teórico, el cual permite comprender lo que es la competitividad ambiental; en la cuarta parte se explica la metodología empleada, para proseguir, en una quinta instancia, con la presentación y el análisis de los resultados, y en la sexta y última parte se entregan las conclusiones.

\section{ISO y la familia de normas ISO 14000}

La ISO es una organización no gubernamental fundada en 1946 con el objetivo de unificar estándares de calidad industrial a nivel mundial. Así, a la fecha, ha publicado 22 
208 estándares internacionales que regulan diversos aspectos. Particularmente, dentro de la familia de normas ISO 14000 se encuentra la 14001, creada en 1996 y que define de manera específica la forma de establecer un SGA. Desde entonces el mundo ha visto un aumento progresivo en el número de empresas certificadas, al pasar de 111163 en 2005 a 346147 en 2016 (ISO, 2018).

Europa merece una mención particular ya que, como lo pronosticaban los estudios (Rothery, 1995), en los primeros años de la ISO 14000 el número de empresas certificadas aumentó de manera considerable al pasar de 3718 en el año 2000 a 8032 en el año 2005, hasta alcanzar un pico de 13889 en 2010. Esta dinámica tendría implicaciones sobre las empresas de otras regiones del mundo que operaban como proveedoras de multinacionales europeas, ya que pronto se verían en la obligación de adoptar la certificación para poder mantener sus tratos comerciales con estas (Miles, Munilla y McClurg, 1999), lo que implicó que productores y proveedores entraran en la dinámica de la certificación.

Sin embargo, en los últimos años lo que se ha visto es una marcada desaceleración en el proceso de certificación de las empresas europeas, lo que puede estar relacionado con el hecho que las compañías establecidas en países desarrollados no se ven sometidas a la misma presión que las provenientes de países en vías de desarrollo. En términos de Tambunlertchai et al. (2013), en estos últimos puede encontrarse una regulación limitada alrededor de los temas ambientales, lo que genera desconfianza por parte de los clientes en el extranjero y desemboca en presiones para la adopción de la ISO 14000, lo que le permite a la empresa ser transparente en la información sobre sus procesos productivos. Según los investigadores, las empresas que reciben inversión extranjera directa (IED) de países de la Organización para la Cooperación y el Desarrollo Económico (OCDE) y de países con mayor número de certificaciones tienen más probabilidades de adoptar la ISO 14001.

El mercado europeo ha demostrado ser más consciente del medio ambiente que aquellos en otras partes industrializadas del mundo. La adopción de certificaciones ofrece así una ventaja valiosa para los productores que desean llegar a los mercados europeos (Bellesi et al., 2005). Para el caso colombiano, vale la pena tener en cuenta que los principales inversionistas provienen de España, Estados Unidos, Canadá e Inglaterra, y que los principales mercados a los que acceden los productos del país son Estados Unidos, Panamá, Holanda, Ecuador y España (Banco de la República, 2018). Como se evidencia, en su mayoría corresponden a países desarrollados en los que la certificación es una garantía de procesos sostenibles.

En Colombia el Instituto Colombiano de Normas Técnicas y Certificación (Icontec) es el órgano encargado de representar al país frente a la ISO. En cuanto a la ISO 14000, la encuesta ISO (2016) demuestra que Colombia es el segundo país de América Latina con empresas certificadas después de Brasil, al alcanzar las 2 993, lo que representa el 28,6 \% del total de la región. Incluso supera a Canadá y México que, aunque son contabilizadas en Norteamérica, son economías superiores en tamaño a la colombiana.

Una de las razones por las cuales las empresas se certifican pensando en obtener beneficios en términos de internacionalización es que, al cumplir con estos estándares, están garantizando a sus clientes que sus productos son seguros y de buena calidad. Esto se traduce en la capacidad de acceder a nuevos mercados en los que la certificación puede ser indispensable para ingresar o simplemente de alcanzar los estándares de calidad que la sociedad exige (ISO, 2018).

Lo anterior concuerda con los resultados de algunas investigaciones (Oliveira, Serra, y Salgado, 2010) según las cuales dentro de los beneficios de implantar un SGA o certificaciones internacionales están: el acceso a nuevos mercados, incentivos, seguros y capital, el cumplimiento de la legislación, la reducción de riesgos y las mejoras en el proceso de producción, el desempeño ambiental, la gestión general de la empresa y la imagen pública, entre otras. Así, en la medida en que las empresas perciban beneficios como resultado de la inserción en estos procesos, más interés se generará por adoptar prácticas amigables con el medio ambiente como una forma de adquirir ventajas competitivas en el mercado.

\section{Fundamento teórico: la competitividad ambiental}

La responsabilidad ambiental implica una serie de retos para las empresas con formas tradicionales de producción que pueden desembocar en el poco interés por establecer nuevas prácticas en las organizaciones. Por lo tanto, para que el discurso de la sostenibilidad se haga realidad, es necesario que la empresa perciba la posibilidad de adquirir beneficios más allá de los ambientales. En ese sentido se encuentran los objetivos de las ISO 14000 y los postulados de la teoría de la competitividad ambiental.

Propuesta por Porter y Van der Linde (1995), la teoría de la competitividad ambiental se ha convertido en el referente teórico por excelencia de las investigaciones que giran alrededor de los beneficios de la implementación de prácticas sostenibles 
en la empresa. La idea consiste en que la sostenibilidad conlleva a que las empresas se hagan más innovadoras con el objetivo de desarrollar procesos o productos que cumplan la normatividad ambiental. Al generar ese potencial innovador, la organización mejora en términos de competitividad en el sentido de que disminuye los costos de producción al ahorrar o hacer un uso eficiente de los recursos, pero además puede hacerse espacio en mercados en los cuales la legislación es más blanda. A este tipo de competitividad los autores la denominan como "Innovation offsets".

Para esta teoría, cuando hay una mejora en general en el desarrollo de los procesos, se provoca lo que llaman "compensaciones por procesos", a través de las cuales se alcanza un mejor desempeño gracias a la aplicación de la normatividad ambiental. En este sentido, para determinar si hay o no beneficios, es indispensable que las empresas conozcan los costos del mal empleo de sus recursos, y de esta forma valorarán la ganancia.

Una vez hecho el cálculo, las empresas tienen entonces la opción de limitarse a hacer lo que la legislación de su país les exige en términos de regulación ambiental - lo que puede interpretarse como una actitud reactiva - o pueden actuar de manera proactiva -que, como definen González-Benito y González-Benito (2006), sucede cuando desde la gerencia se asume de forma voluntaria el reto de ir más allá de lo requerido por la ley-. La búsqueda y obtención de certificaciones internacionales puede ser entendida como una estrategia proactiva. Estudios como los de Martín-Tapia, Aragón-Correa y Senise-Barrio (2008) han logrado demostrar que la proactividad ambiental puede influenciar de manera positiva el desempeño exportador. Sin embargo, para que estas iniciativas sean exitosas, es necesario contar con directivos realmente comprometidos.

Leonidou et al. (2015) corroboran la hipótesis propuesta en su estudio respecto a que la alta gerencia de la empresa debe estar involucrada para que así mismo haya una exitosa aplicación de prácticas verdes. Los autores también afirman que la adopción de estas puede generar una ventaja diferenciadora en mercados internacionales y mejorar así el desempeño exportador de la empresa. Esto indica que se depende de la existencia de lo que se ha denominado como un liderazgo ambiental, asociado a prácticas administrativas, para que se aplique un sistema de gestión ambiental certificado o cualquier tipo en general de políticas ambientales proactivas y exitosas.

\section{METODOLOGÍA}

Teniendo en cuenta que el objetivo es identificar si existen diferencias en el rendimiento exportador entre empresas certificadas y no certificadas con ISO 14001, se realizó un estudio de corte transversal de los datos de exportación para cada caso. En este sentido, se solicitó a las entidades certificadoras de Colombia, reconocidas por el Organismo Nacional de Acreditación de Colombia (ONAC), la información sobre las empresas certificadas con ISO 14001 en el país. De las ocho certificadoras, se recibió respuesta de dos: Icontec y Future Builders Colombia S. A. U. E. N MSC, y así se obtuvo la razón social de 1078 empresas.

Posteriormente, con el dato de la razón social, se indagó en la plataforma del Registro Único Empresarial (RUE) sobre el NIT, la ubicación geográfica y la actividad económica de cada una de las empresas suministradas por las certificadoras. De este modo se obtuvo información de 772 organizaciones, es decir, del 71,6 \%. Para la información de activos, a fin de clasificar las empresas por tamaño, se utilizó la base de datos Legiscomex, en la cual se logró encontrar cifras de 111 empresas, es decir, 10,3\% del total suministrado por las certificadoras.

De otro lado, para indagar si existe diferencia en el rendimiento exportador entre empresas certificadas y no certificadas, se tomaron los datos de exportación de empresas colombianas en el primer trimestre del año 2018 del Departamento Administrativo Nacional de Estadística (DANE). Este grupo de empresas fue clasificado en certificadas y no certificadas con ISO 14001 (Tambunlertchai et al., 2013).

Finalmente, para ver la diferencia entre el rendimiento exportador de estos dos grupos de empresas, se realizó una prueba de hipótesis no paramétrica debido a que la distribución de los datos para los dos casos resultó no normal. De esta manera, se usó la prueba U de Mann-Whitney en la cual se busca contrastar la mediana de los datos.

\section{RESULTADOS}

Como se mencionó anteriormente, de las 1078 razones sociales de firmas certificadas con ISO 14001, se encontraron datos de activos, actividad económica y ubicación geográfica para el 10,3\%, muestra que concuerda con estudios similares (Seijo-García et al., 2013; Tambunlertchai et al, 2013; Wysokińska y Witkowska, 2005). De estas empresas, el 64,9 $\%$ son grandes; $25,2 \%$, medianas; $9 \%$, pequeñas, y $0,9, \%$ microempresas. Si se analiza esta categorización por regiones, se encuentra que la mayor parte de las grandes empresas certificadas se ubica en Bogotá (31,9\%), Antioquia (16,6 \%), Atlántico $(9,7 \%)$ y Valle del Cauca $(9,7 \%)$. Las 
empresas medianas se ubican en su mayoría en Bogotá (46,4 $\%)$, Antioquia $(14,3 \%)$ y Santander (14,3\%). Las micro y pequeñas empresas se distribuyen de manera similar en las regiones analizadas.

Respecto a la actividad económica de las empresas certificadas, la figura 1 muestra los porcentajes de distribución, en los que se evidencia que industria manufacturera, construcción y actividades profesionales, científicas y técnicas, que se refiere en esencia a empresas asesoras de obras de ingeniería civil y arquitectura, son las que más recurren a certificarse en la norma ISO 14001.

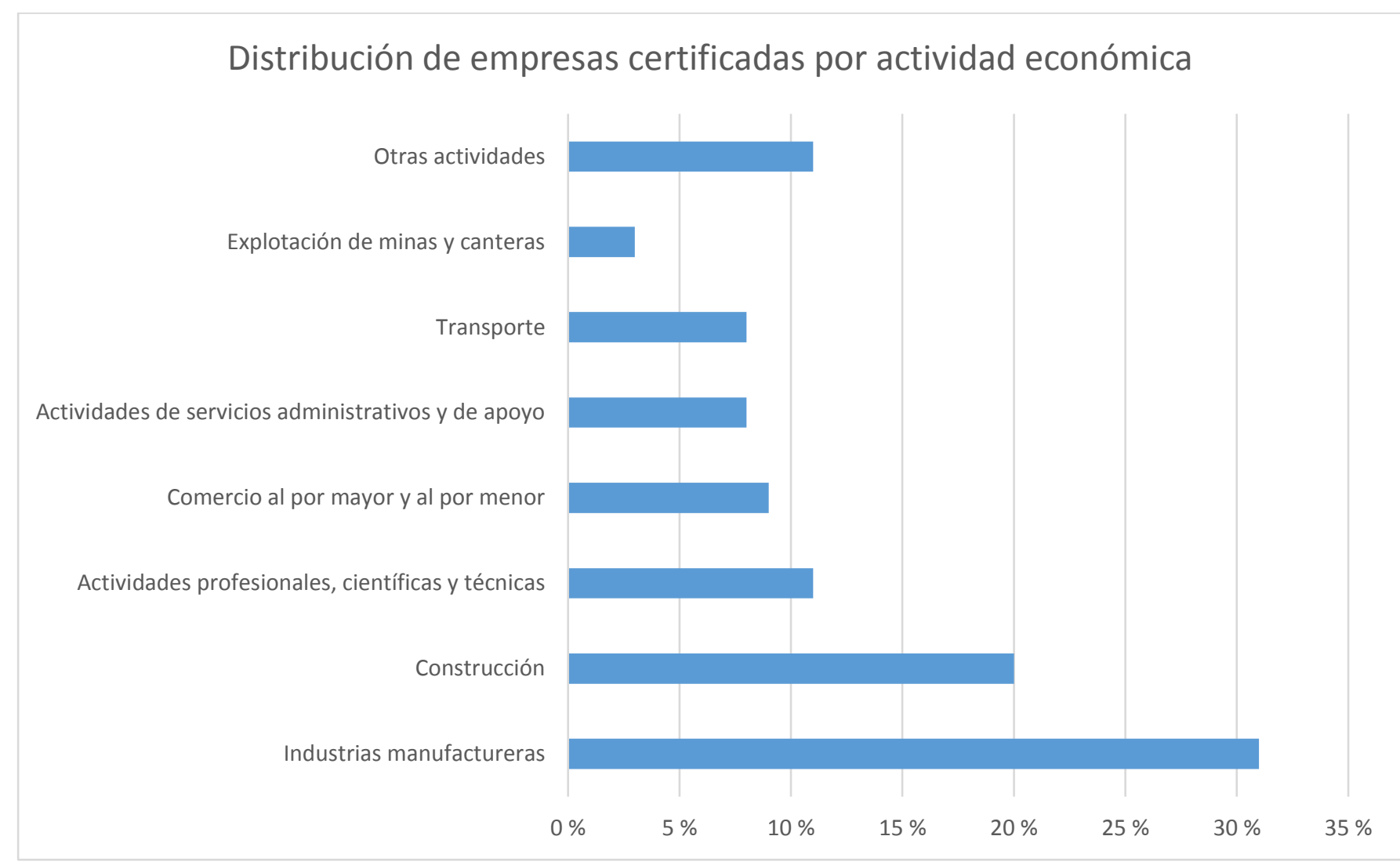

Figura 1. Distribución de empresas certificadas por actividad económica.

Fuente: elaboración propia.

En cuanto a la ubicación geográfica, Bogotá, Antioquia y Santander son las regiones con mayor presencia de empresas certificadas, lo que es comprensible ya que estas son importantes centros industriales del país.

Retomando el objetivo del estudio, se pretendía establecer si hay diferencia en el volumen exportador entre una empresa certificada y una no certificada. Para ello se tomaron los datos de exportación de Colombia en el primer trimestre del año 2018 y se discriminó, conforme a la base obtenida de Icontec, entre empresas certificadas y no certificadas. De tal modo se encontró que, de las 6008 empresas que exportaron en dicho período, 124 están certificadas con la norma ISO 14001.

Al realizar el análisis de los datos de exportación de empresas certificadas y no certificadas, se encontró que estos no tienen distribución normal, como lo evidencian la tabla 1 (descriptivos para empresas certificadas) y la tabla 2 (descriptivos para empresas no certificadas). 
Tabla 1. Descriptivos para empresas certificadas.

\begin{tabular}{|c|c|c|c|c|}
\hline \multirow{2}{*}{\multicolumn{3}{|c|}{\begin{tabular}{|l|l|}
\multicolumn{2}{|c|}{} \\
Fxnortaciones & Media
\end{tabular}}} & \multirow{2}{*}{\begin{tabular}{|l|} 
Estadístico \\
$2,2934 \mathrm{E} 7$ \\
\end{tabular}} & \multirow{3}{*}{\begin{tabular}{|l} 
Error típ. \\
$1,61462 \mathrm{E} 7$ \\
\end{tabular}} \\
\hline & & & & \\
\hline & \multirow{2}{*}{$\begin{array}{l}\text { Intervalo de confianza para la } \\
\text { media al } 95 \%\end{array}$} & Límite inferior & $-9,0263 \mathrm{E} 6$ & \\
\hline & & Límite superior & 5,4894E7 & \\
\hline & \multicolumn{2}{|l|}{ Media recortada al $5 \%$} & $3,4034 \mathrm{E} 6$ & \\
\hline & \multicolumn{2}{|l|}{ Mediana } & 933492,4050 & \\
\hline & \multicolumn{2}{|l|}{ Varianza } & 3,233E16 & \\
\hline & \multicolumn{2}{|l|}{ Desv. típ. } & $1,79796 \mathrm{E} 8$ & \\
\hline & \multicolumn{2}{|l|}{ Mínimo } & 150,00 & \\
\hline & \multicolumn{2}{|l|}{ Máximo } & $2,00 \mathrm{E} 9$ & \\
\hline & \multicolumn{2}{|l|}{ Rango } & $2,00 \mathrm{E} 9$ & \\
\hline & \multicolumn{2}{|l|}{ Amplitud intercuartil } & 4017213,87 & \\
\hline & \multicolumn{2}{|l|}{ Asimetría } & 10,947 & 0,217 \\
\hline & \multicolumn{2}{|l|}{ Curtosis } & 121,096 & 0,431 \\
\hline
\end{tabular}

Fuente: elaboración propia.

Tabla 2. Descriptivos para empresas no certificadas.

\begin{tabular}{|c|c|c|c|c|}
\hline & & & Estadístico & Error típ. \\
\hline \multirow[t]{13}{*}{ Exportaciones } & \multicolumn{2}{|l|}{ Media } & $1,1576 \mathrm{E} 6$ & $1,73332 \mathrm{E} 5$ \\
\hline & \multirow{2}{*}{$\begin{array}{l}\text { Intervalo de confianza para la } \\
\text { media al } 95 \%\end{array}$} & Límite inferior & 817831,1238 & \\
\hline & & Límite superior & 1,4974E6 & \\
\hline & \multicolumn{2}{|l|}{ Media recortada al $5 \%$} & 167584,8181 & \\
\hline & \multicolumn{2}{|l|}{ Mediana } & 30261,6250 & \\
\hline & \multicolumn{2}{|l|}{ Varianza } & $1,768 \mathrm{E} 14$ & \\
\hline & \multicolumn{2}{|l|}{ Desv. típ. } & $1,32958 \mathrm{E} 7$ & \\
\hline & \multicolumn{2}{|l|}{ Mínimo } & 1,00 & \\
\hline & \multicolumn{2}{|l|}{ Máximo } & $5,49 \mathrm{E} 8$ & \\
\hline & \multicolumn{2}{|l|}{ Rango } & $5,49 \mathrm{E} 8$ & \\
\hline & \multicolumn{2}{|l|}{ Amplitud intercuartil } & 153816,33 & \\
\hline & \multicolumn{2}{|l|}{ Asimetría } & 28,267 & 0,032 \\
\hline & \multicolumn{2}{|l|}{ Curtosis } & 947,300 & 0,064 \\
\hline
\end{tabular}

Fuente: elaboración propia.

Conforme a estos datos, se realizó una prueba no paramétrica U de Mann-Whitney. El resultado lleva a rechazar la hipótesis de igualdad de las medianas, lo que implica que hay una diferencia estadística significativa entre estas medidas. Así pues, se acepta la hipótesis de que existe diferencia entre las medianas del volumen de exportaciones entre empresas 
Tabla 3. Estadísticos de contraste.

\begin{tabular}{|l|l|}
\hline & Exportaciones \\
\hline U de Mann-Whitney & 150120,000 \\
W de Wilcoxon & $1,746 \mathrm{E} 7$ \\
Z & $-11,232$ \\
Sig. asintót. (bilateral) & 0,000 \\
& \\
\hline
\end{tabular}

a. Variable de agrupación: certificación

Fuente: elaboración propia.

Conforme a los estadísticos descriptivos para los grupos de empresas certificadas y no certificadas que se muestran en las tablas 1 y 2, los datos no se comportan de una manera normal. Esto quiere decir que la variable exportaciones es muy volátil, lo cual se puede deber a distintas causas, es decir, no necesariamente está relacionado directamente con el hecho de ser una empresa certificada o no. Lo que sí se puede decir es que los resultados muestran que las empresas certificadas tienen en promedio una tendencia a tener un mayor volumen de exportación que las que no están certificadas. Sin embargo, es necesario aclarar que no se midió la correlación debido a la falta de información.

\section{DISCUSIÓN}

Con base en los resultados se puede afirmar que, en el grupo de empresas estudiadas, aquellas con certificación perciben un mayor volumen exportador que las que no tienen certificación. Sin embargo, es necesario tener en cuenta que el grupo de empresas colombianas certificadas corresponde en su mayoría ( $70 \%$ ) a compañías grandes, consolidadas en el mercado nacional y en algunos casos con sólidos procesos de internacionalización, concordando con lo aportado por Aguilera et al. (2012). En este sentido, estas variables deben entrar en consideración en estudios posteriores.

Los resultados obtenidos para el caso colombiano contradicen los presentados en las investigaciones de Tambunlertchai et al. (2013) y Bellesi et al. (2005), quienes, aunque reconocen la importancia de la certificación, no logran identificar el impacto de esta -0 su relación- sobre las exportaciones. Por el contrario, sí se encuentra similitud con Luan et al. (2016) y Aguilera et al. (2012) en el sentido de que hay relación entre el nivel de internacionalización y la adopción de certificaciones. Como se puede apreciar, se confirma la diversidad en los resultados obtenidos en este tipo de investigaciones identificada por Chen et al. (2016).

Teniendo en cuenta que los resultados encontrados en la presente investigación confirmarían que la estrategia funciona, ya que se evidencia que las empresas certificadas están exportando más que las no certificadas, se podría deducir que hay coincidencia con Bellesi et al. (2005) cuando menciona que este tipo de certificación es indispensable para las empresas que operan en países en desarrollo. Asimismo, como lo recalcan Pekovic y Rolland (2016) y Seijo et al. (2013), la certificación se convierte en un elemento reputacional que permite comunicarle al cliente los estándares de calidad ambiental adoptados.

Sin embargo, es necesario tener en cuenta las limitaciones del estudio en el sentido de que se tomaron datos de corte transversal correspondientes a las exportaciones del primer trimestre del año 2018, lo cual lleva a que hayan sido observadas solo las empresas certificadas que efectivamente exportaron en ese período, descartando así algunas empresas que por diferentes motivos no hayan exportado en el momento de la observación. De otro lado, como se describió en los resultados, los descriptivos de los datos de exportación evidenciaron que, tanto para el grupo de empresas certificadas como las no certificadas, estos tienen alta volatilidad lo cual no puede asociarse a la condición de certificación ISO 14001; para ello requeriría hacerse pruebas de correlación con otras variables que incidan en el desempeño exportador. En estudios posteriores se podrían 
establecer dichas correlaciones para medir el impacto de aplicar estrategias verdes en las empresas colombianas.

\section{CONCLUSIÓN}

La necesidad de llevar a la práctica el discurso ambiental ha llevado a las empresas a adoptar diferentes tipos de estrategias con distintos niveles de complejidad dependiendo de su actividad económica, capacidad financiera, mercado al que se dirigen y sus exigencias, entre otras. Sin embargo, una manera efectiva de hacer visibles estas prácticas y el compromiso con la sostenibilidad, generando además beneficios para la organización a partir de ello, es la obtención de certificaciones como la ISO 14000. Si bien las empresas que incursionan en este tipo de proyectos deben estar preparadas para asumir los costos, es probable que tomen el riesgo esperando adquirir algún tipo de beneficio o ventaja en el mercado.

Una de las ventajas de la ISO es su carácter internacional. $\mathrm{Al}$ ser reconocida en 161 países alrededor del mundo, los productos avalados por la organización serán reconocidos en estos mercados por cumplir con los estándares de calidad. En el caso específico de la ISO 14000, la garantía que se brinda es la de la aplicación de un sistema de gestión ambiental, con lo que ello implica. Como se ha explicado en el texto, en mercados con consumidores exigentes, sobre todo en países desarrollados, la certificación es una ventaja más cuando el producto proviene de países en vía de desarrollo donde la legislación ambiental es más flexible, de manera que la única forma de asegurar que se ha hecho un buen uso de los recursos es a través de la certificación. En este orden de ideas, resulta lógico que las empresas con mayor nivel de internacionalización o con el objetivo de aumentar su acceso a mercados en el extranjero buscarán la certificación, lo que aplica principalmente a empresas grandes.

Frente a este contexto, las empresas colombianas han respondido de manera positiva a los nuevos retos de la sostenibilidad. Así lo sugiere el incremento de compañías certificadas con ISO 14001 en el país, respondiendo a la dinámica internacional en la que, ante todo, las empresas grandes e internacionalizadas son las más interesadas en ser certificadas para no poner en riesgo sus mercados en el extranjero y aumentar su participación internacional. El campo de investigación en la materia es amplio, y su profundización puede contribuir a aumentar el interés de las compañías por hacer de la sostenibilidad una estrategia de competitividad.

\section{Declaración sobre conflicto de interés}

Los autores del presente artículo declaramos que somos independientes de la institución que ha financiado el estudio y, por tal razón, durante la ejecución de la investigación y la redacción del documento no han incidido intereses diferentes a los académicos.

\section{REFERENCIAS BIBLIOGRÁFICAS}

Aguilera-Caracuel, J., Hurtado-Torres, N. E. y AragónCorrea, J. A. (2012). Does international experience help firms to be green? A knowledge-based view of how international experience and organizational learning influence proactive environmental strategies. International Business Review, 21(5), 847-861. doi:

https://doi.org/10.1016/j.ibusrev.2011.09.009

Antonietti, R. y Marzucchi, A. (2014). Green tangible investment strategies and export performance: a firm-level investigation. Ecological Economics, 108, 150-161. doi: https://doi.org/10.1016/j.ecolecon.2014.10.017

Banco de la República. (2018). Flujo de Inversión Extranjera Directa según país de destino. Recuperado de http://www.banrep.gov.co/es/inversion-directa

Bellesi, F., Lehrer, D. y Tal, A. (2005). Comparative Advantage: The Impact of ISO 14001 Environmental Certification on Exports. Environ. Sci. Technol., 39(7), 1943-1953. doi: https://doi.org/https://doi.org/10.1021/es0497983

Clarkson, P., Li, Y., Richardson, G. y Vasvari, F. P. (2011). Does it really pay to be green? 
Determinants and consequences of proactive environmental strategies. Journal of Accounting and Public Policy, 30(2), 122-144. Recuperado de https://econpapers.repec.org/article/eeejappol/v_3 a30_3ay_3a_3ai_3a2_3ap_3a122-144.htm

Chen, P. H., Ong, C. F. y Hsu, S. C. (2016). Understanding the relationships between environmental management practices and financial performances of multinational construction firms. Journal of Cleaner Production, 139, 750-750. doi: https://doi.org/10.1016/j.jclepro.2016.08.109

Dragičević, M. y Letunić, S. (2012). The influence of international standards ISO 9001 and ISO 14001 on marketing performances of Croatian hotels. Tourism \& Hospitality Management, Conference Proceedings, 22(1), 102-110. Recuperado de https://www.daaam.info/Downloads/Pdfs/proceed ings/proceedings_2011/0265_Dragicevic.pdf

Feng, T., Cai, D., Wang, D. y Zhang, X. (2016). Environmental management systems and financial performance: the joint effect of switching cost and competitive intensity. Journal of cleaner production, 113, 781-791. doi: https://doi.org/10.1016/j.jclepro.2015.11.038

González-Benito, J. y González-Benito, O. (2006). A review of determinant factors of environmental proactivity. Business Strategy and the Environment, 15(2), 87-102. doi: https://doi.org/10.1002/bse.450
González, P., Sarkis, J. y Adenso-Díaz, B. (2008). Environmental management system certification and its influence on corporate practices: Evidence from the automotive industry. International Journal of Operations \& Production Management, 28(11), 1021-1041. doi: https://doi.org/10.1108/01443570810910179

Jänicke, M. (2008). Ecological modernization: new perspectives. Journal of Cleaner Production, 16(5), 557-565. doi: https://doi.org/10.1016/j.jclepro.2007.02.011

King, A., Lenox, M. y Terlaak, A. (2005). The strategic use of decentralized institutions: Exploring certification with the ISO 14001 management standard. Academy of Management Journal, 48(6), 1091-1106. doi: https://doi.org/10.5465/amj.2005.19573111

Leonidou, C. L., Fotiadis, T. A., Christodoulides, P., Spyropoulou, S. y Katsikeas, C. S. (2015). Environmentally friendly export business strategy: Its determinants and effects on competitive advantage and performance. International Business Review, 24(5), 798-811. doi: https://doi.org/10.1016/j.ibusrev.2015.02.001

Lo, C. K., Yeung, A. C. y Cheng, T. C. (2012). The impact of environmental management systems on financial performance in fashion and textiles industries. International Journal Production Economics, Elsevier, 135(2), 561-567. Recuperado de 
https://ideas.repec.org/a/eee/proeco/v135y2012i2 p561-567.html

Lucas, M. T. y Noordewier, T. G. (2016). Environmental management practices and firm financial performance: The moderating effect of industry pollution-related factors. International Journal Production Economics, Elsevier, 175, 24-34. doi: https://doi.org/10.1016/j.ijpe.2016.02.003

Luan, C. J., Tien, C. y Chen, W. L. (2016). Which "green" is better? An empirical study of the impact of green activities on firm performance. Asia Pacific Management Review, 21(2), 102-110. doi: https://doi.org/10.1016/j.apmrv.2015.12.001

Martín-Tapia, I., Aragón-Correa, J. y Senise-Barrio, M. (2008). Being green and export intensity of SMEs: The moderatinginfluence of perceived uncertainty. Ecological Economics, Elsevier, 68(1-2), 56-67. doi:

https://doi.org/10.1016/j.ecolecon.2008.01.032

Miles, M. P., Munilla., S. L. y McClurg, T. (1999). The impact of the ISO 14000 environmental management standards on small and medium sized enterprises. Journal of Quality Management, 4(1), 111-122. doi: https://doi.org/10.1016/S1084$\underline{8568(99) 80098-4}$

Miroshnychenko, I., Barontini, R. y Testa, F. (2017). Green practices and financial performance: A global outlook. Journal of Cleaner Production,
147 ,

340-351.

doi: https://doi.org/10.1016/j.jclepro.2017.01.058

Neves, F. O., Salgado, E. y Beijo, L. (2017). Analysis of the Environmental Management System based on ISO 14001 on the American continent. Journal of Environmental Management, 199, 251-262. doi: https://doi.org/10.1016/j.jenvman.2017.05.049

Oliveira, O. J., Serra, J. R. y Salgado, M. (2010). Does ISO 14001 work in Brazil? Journal of Cleaner Production, 18(18), 1797-1806. doi: https://doi.org/10.1016/j.jclepro.2010.08.004

Organización Internacional para la Estandarización - ISO. (2018). When the world agrees. Recuperado de https://www.iso.org/files/live/sites/isoorg/files/sto re/en/PUB100007.pdf

Organización Internacional para la Estandarización - ISO. (2016). The ISO Survey of Management System Standard Certifications (1999-2016). Recuperado de

https://isotc.iso.org/livelink/livelink?func=11\&objI $\mathrm{d}=18808772 \&$ objAction=browse \&viewType $=1$

Pekovic, S. y Rolland, S. (2016). Quality standards and export activities: Do firm size and market destination matter? Journal of High Technology Management Research, 27, 110-118. doi: https://doi.org/10.1016/j.hitech.2016.10.002

Porter, M. y Van der Linde, C. (1995). Toward a new conception of the environment-competitiveness relationship. The Journal of the Economic 
¿INCIDE LA ISO 14000 EN EL DESEMPEÑO EXPORTADOR DE LAS EMPRESAS COLOMBIANAS?

Perspectives, 9(4), 97-118. doi: http://dx.doi.org/10.1257/jep.9.4.97

Psomas, E., Fotopoulos, C. y Kafetzopoulos, D. (2011). Motives, difficulties and benefits in implementing the ISO 14001 Environmental Management System. Management of Environmental Quality: An International Journal, 22(4), 502-521. doi: https://doi.org/10.1108/14777831111136090

Rothery, B. (1995). Why ISO 14000 will catch ISO 9000. Manufacturing Engineering, 115(5), 128.

Rowland-Jones, R., Pryde, M. y Cresser, M., (2005). An evaluation of current environmental management systems as indicators of environmental performance. Management of Environmental Quality: An International Journal, 16(3), 211-219. doi: https://doi.org/10.1108/14777830510591642

Seijo-García, M., Filgueira-Vizoso, A. y MuñozCamacho, E. (2013). Consecuencias positivas de la implantación de la certificación ISO 14001 en las empresas Gallegas (España). Dyna, 80(177), 13-21. Recuperado de https://revistas.unal.edu.co/index.php/dyna/article /view/34035

Tambunlertchai, K., Kontoleon, A. y Khanna, M. (2013). Assessing Participation in Voluntary Environmental Programmes in the Developing World: The Role of FDI and Export Orientation on ISO14001 Adoption in Thailand. Applied Economics, 45(15), 2039-2048. doi: https://doi.org/10.1080/00036846.2011.648320

Wysokińska, Z. y Witkowska, J. (2005). International Business and Environmental Issues - Some Empirical Evidence from Transition Economies. Polish Journal of Environmental Studies, 14(3), 269-279. Recuperado de http://www.pjoes.com/International-Businessand-Environmental-Issues-Some-EmpiricalEvidence-from-Transition,87757,0,2.html 